\title{
A Case of Repeated TAFRO Syndrome-Like Symptoms and Retroperitoneal Hemorrhage in a Patient With Sjögren Syndrome
}

\author{
Takanori Ohta ${ }^{1}$, Naoki Oda ${ }^{1}$, Keiko Saito ${ }^{1}$, Sadafumi Tamiya ${ }^{2}$, Toshiyuki Ueno ${ }^{1}$ \\ 1. Department of Internal Medicine, Kitakyushu Municipal Medical Center, Kitakyushu, JPN 2. Department of \\ Pathology, Kitakyushu Municipal Medical Center, Kitakyushu, JPN
}

Corresponding author: Takanori Ohta, t_oota01@kmmc.jp

\begin{abstract}
A 50-year-old Japanese man complaining of dry mouth and eyes, pale skin with cold irritation, and worsening epigastric pain was admitted to the hospital, whereupon he developed fever and anasarca. A computed tomography (CT) scan showed ascites, hepatosplenomegaly, and mildly enlarged multiple lymph nodes, and blood examination revealed renal impairment, thrombocytopenia, and high levels of C-reactive protein (CRP). He was diagnosed with Sjögren syndrome and concurrently manifested symptoms resembling TAFRO syndrome (i.e., thrombocytopenia (T), anasarca (A), fever (F), reticulin fibrosis (R), and organomegaly (O)). Although the TAFRO syndrome-like symptoms progressed, he gradually recovered with immunosuppressive agents. Seven years and five months after the admission, the TAFRO syndrome-like symptoms recurred. Bone marrow biopsy specimens revealed reticulin fibrosis. Inguinal and mediastinal lymph nodes biopsy specimens revealed Castleman disease-like features. Although the symptoms indicated TAFRO syndrome, a diagnosis was not possible owing to the presence of hypergammaglobulinemia and Sjögren syndrome, which required exclusion. Corticosteroid treatment was initiated; however, it was complicated by retroperitoneal hemorrhage, probably due to microangiopathy. After additional treatment with tocilizumab and rituximab, the TAFRO syndrome-like symptoms improved and the hemorrhage progression stopped. In conclusion, TAFRO syndrome-like symptoms may recur with vascular complications and can be successfully treated with tocilizumab and rituximab during Sjögren syndrome. The etiology of TAFRO syndrome could potentially involve Sjögren syndrome, and these syndromes may co-exist.
\end{abstract}

Received 05/21/2020 Review began 06/09/2020 Review ended 12/13/2020 Published 12/19/2020

\section{() Copyright 2020}

Ohta et al. This is an open access article distributed under the terms of the Creative Commons Attribution License CC-BY 4.0., which permits unrestricted use, distribution, and reproduction in any medium, provided the original author and source are credited.
Categories: Internal Medicine, Rheumatology, Hematology

Keywords: tafro syndrome, sjögren syndrome, microangiopathy, tocilizumab, rituximab, tma, multicentric castleman disease, autoimmune diseases, retroperitoneal hemorrhage

\section{Introduction}

TAFRO syndrome is a recently recognized systemic inflammatory disorder, characterized by thrombocytopenia $(\mathrm{T})$, anasarca $(\mathrm{A})$, fever $(\mathrm{F})$, reticulin fibrosis $(\mathrm{R})$, and organomegaly $(\mathrm{O})$. Its diagnostic criteria, disease severity classification, and treatment strategy were proposed by Masaki et al. in 2015 [1]. According to the updated 2019 diagnostic criteria for TAFRO syndrome, Sjögren syndrome has been newly added to the exclusion criteria during differential diagnosis [2]. However, patients with a definitive diagnosis of Sjögren syndrome or those positive for Sjögren's antibodies but with an uncertain diagnosis have reportedly manifested the clinicopathological features of TAFRO syndrome [3-7]. Cases of human herpes virus-8-negative multicentric Castleman disease (MCD) of unknown etiology are referred to as idiopathic MCD (iMCD). Positive cases of anti-Sjögren's syndrome-related antigen A (anti-SS-A) antibody have been reported in iMCD, of which TAFRO-iMCD syndrome is considered a subtype [8-10]. Thus, TAFRO syndrome could be associated with Sjögren syndrome. However, the relationship between these two syndromes is still unclear and its differential diagnosis remains challenging. Therefore, this case, which presented repeated TAFRO syndrome-like symptoms and hemorrhage related to thrombotic microangiopathy (TMA) during Sjögren syndrome, provides valuable information about the association between and etiology of the two syndromes.

\section{Case Presentation}

A 50-year-old Japanese man with a medical history of sinusitis and hyperuricemia complained of dry mouth and eyes from September 2011, and pale skin on cold irritation and epigastric pain from December 2011. Because over-the-counter medications were not effective for the progressing epigastric pain, he visited a neighboring general hospital in January 2012 where a computed tomography (CT) scan showed mildly enlarged mediastinal and aortic lymphadenopathy, and blood examination showed elevated creatinine (Cre) level (1.39 mg/dL), thrombocytopenia $\left(9 \times 10^{4} / \mu \mathrm{L}\right)$, and a high level of C-reactive protein (CRP) $(13.05 \mathrm{mg} / \mathrm{dL})$. Considering that no apparent gastrointestinal, cardiac, or infectious disease could be identified, as well as the presence of dryness symptoms and Raynaud's phenomenon, the patient was referred to a department of rheumatology in a larger general hospital. After admission to the larger hospital in February 2012, he developed a high fever. His physical examination revealed abdominal tenderness, anasarca, dry mouth and 


\section{Cureus}

eyes, and symptoms typical of Raynaud's phenomenon. The results of the laboratory examinations are presented in Table 1 .

\begin{tabular}{|c|c|c|c|}
\hline \multicolumn{2}{|l|}{ Situation } & onset & relapse \\
\hline & Admission year & 2012 & 2019 \\
\hline \multicolumn{4}{|c|}{ Blood examinations (reference range) } \\
\hline & White blood cells, $\mu \mathrm{L}$ & 8700 & 3000 \\
\hline & Neutrophils, \% & 81 & 27 \\
\hline & Lymphocytes, \% & 10 & 53 \\
\hline & Monocytes, \% & 9 & 13 \\
\hline & Eosinophils, \% & 0 & 5 \\
\hline & Basophils, \% & 0 & 2 \\
\hline & Red blood cells, $10^{6} / \mu \mathrm{L}$ & 502 & 305 \\
\hline & Reticulocytes, \% & 6 & 4 \\
\hline & Hemoglobin, g/dL & 15.8 & 9.9 \\
\hline & Platelets, $10^{4} / \mu \mathrm{L}$ & 8.1 & 4.4 \\
\hline & Minimum platelet count & 0.3 & 1.9 \\
\hline & Aspartate aminotransferase, IU/L & 19 & 12 \\
\hline & Alanine aminotransferase, IU/L & 18 & 4 \\
\hline & Lactate dehydrogenase, IU/L & 236 & 145 \\
\hline & Alkaline phosphatase, IU/L & 1084 & 436 \\
\hline & v-glutamyl transpeptidase, IU/L & 188 & 31 \\
\hline & Total protein, mg/dL & 6.5 & 7.6 \\
\hline & Albumin, mg/dL & 3 & 3.4 \\
\hline & Total-bilirubin, mg/dL & 0.6 & 1.1 \\
\hline & Creatinine, mg/dL & 1.04 & 1.11 \\
\hline & Maximum creatinine value & 5.83 & 3.79 \\
\hline & Blood urea nitrogen, $\mathrm{mg} / \mathrm{dL}$ & 27 & 19.4 \\
\hline & Creatine kinase, IU/L & 21 & 31 \\
\hline & Ferritin, ng/mL & 218 & 297.9 \\
\hline & C-reactive protein, mg/dL & 25.72 & 1.282 \\
\hline & Maximum c-reactive protein value & 29.47 & 2.318 \\
\hline & IgG, mg/dL & 1024 & 2500 \\
\hline & $\operatorname{lgA}, \mathrm{mg} / \mathrm{dL}$ & 214 & 329 \\
\hline & $\operatorname{lgM~mg/dL~}$ & 244 & 337 \\
\hline & Anti-nuclear antibody & $<40$ & $<40$ \\
\hline & Anti-SS-A* antibody, U/mL & negative & negative \\
\hline & Anti-SS-B antibody, U/mL & negative & negative \\
\hline & Anti-mitochondrial M2 antibody (<7) & 181 & 551 \\
\hline & Rheumatoid factor, IU/mL & negative & negative \\
\hline
\end{tabular}




\section{Cureus}

Anti-DNA antibody, IU/mL

NA*

Anti-double-stranded DNA antibody

Anti-Smith antibody

Platelet associated lgG, $\mathrm{ng} / 10^{7}$ cells

Direct Coombs test

Indirect Coombs test

Lupus anticoagulant

Anti-cardiolipin antibody

MPO-ANCA*

PR3-ANCA*

SIL-2R* U/mL (<534)

$\mathrm{IL}-6^{*}, \mathrm{pg} / \mathrm{mL}(<4)$

VEGF $^{\star}, \mathrm{pg} / \mathrm{mL}$

HHV-8* DNA

HIV* antibody

$\mathrm{CMV}^{*}-\operatorname{IgM}(<0.8)$

CMV-IgG (<2)

CMV antigenemia

EBV* capsid antigen-lgG $(<10)$

EBV capsid antigen- $\operatorname{lgM}(<10)$

EBV nuclear antigen $(<10)$

Urine examinations

Protein

Occult blood

ß2-microglobulin, $\mathrm{ng} / \mathrm{mL}$

237

1148

NA

NA

NA

NA

NA

20

$<10$

40

negative

negative

negative

negative

237

negative

negative

negative

negative

negative

negative

negative

negative

negative

negative

negative

negative

1360

15.1

70

negative

negative

negative

2.01

negative

negative

NA

$<10$

NA

$1+$

negative

2257
$2+$

$2+$

5698.3

\section{TABLE 1: Laboratory findings at onset and relapse}

Abbreviations: Anti-SS-A/B: Anti-Sjögren's-syndrome-related antigen A/B, NA: not analyzed, MPO-ANCA: Myeloperoxidase-anti neutrophil cytoplasmic antibody, PR3-ANCA: Proteinase 3 anti-neutrophil cytoplasmic antibody, SIL-2R: Soluble interleukin-2 receptor, IL-6: Interleukin-6, VEGF: Vascular endothelial growth factor, HHV-8: Human herpesvirus-8, HIV: Human immunodeficiency virus, CMV: Cytomegalovirus, EBV: EpsteinBarr virus

A CT scan showed ascites, hepatosplenomegaly, and mildly enlarged lymph nodes in the inguinal, mediastinal, and para-aortic regions. Inguinal lymph node biopsy specimens did not provide a definitive diagnosis such as malignant lymphoma. No infectious diseases responsible for the elevated CRP could be clinically detected. Lip biopsy of his labial salivary gland showed focal lymphocytic sialadenitis and a focus score $\geqslant$ of $1 \mathrm{foci} / 4 \mathrm{~mm}^{2}$, and the unstimulated whole saliva flow rate was $0.08 \mathrm{~mL} / \mathrm{minute}$. Based on those findings, a diagnosis of Sjögren syndrome was made, although antibodies for anti-SS-A and anti-Sjögren'ssyndrome-related antigen B (anti-SS-B) were negative. In addition, the patient had symptoms of dry mouth and eyes lasting six months, and no disease other than Sjögren syndrome was identified as causing the sicca syndrome. Increased levels of alkaline phosphatase (ALP) and positivity for the anti-mitochondrial M2 antibody were related to subclinical primary biliary cholangitis. These symptoms could be explained as an acute exacerbation of Sjögren syndrome with subclinical primary cholangitis. However, the patient's symptoms exhibited aggressive clinical behavior compared to the typical course of Sjögren syndrome. Therefore, complications of other autoimmune diseases, such as vasculitis, were suspected but not identified. Corticosteroid pulse followed by high-dose corticosteroid was administered (starting from day 1) as first-line therapy, but it had no dramatic impact on the patient's epigastric pain, anasarca, fever, or 


\section{Cureus}

abnormal blood results, including the CRP level, Cre level, and thrombocytopenia. Furthermore, pleural effusion, which had serous characteristics without pathogens and atypical cells, appeared and increased. Dialysis for progressive anasarca and renal dysfunction was initiated on day 6, as shown in Figure 1.

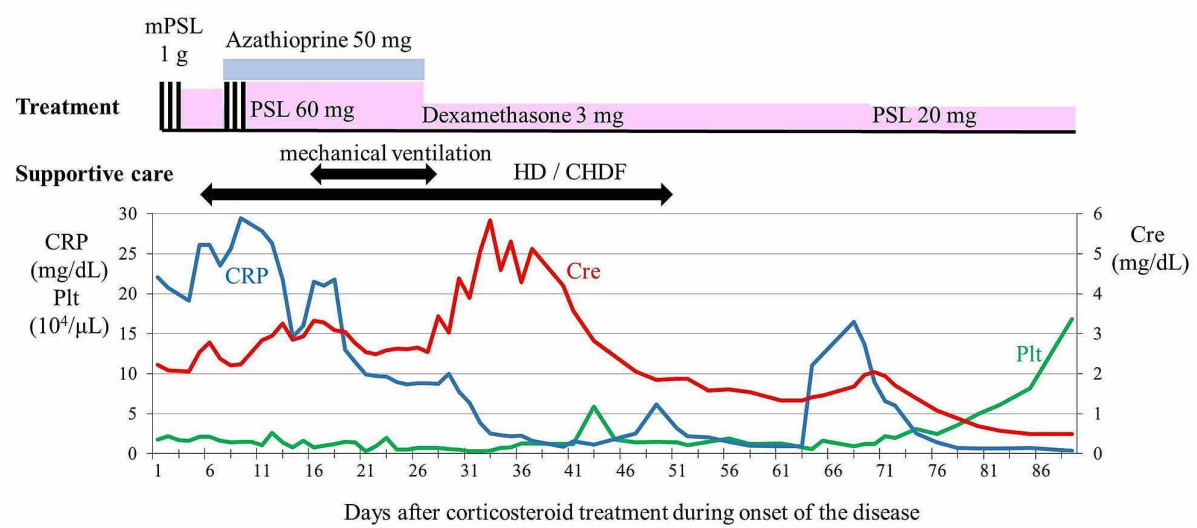

FIGURE 1: Clinical course of onset

Abbreviations: mPSL: Methylprednisolone, PSL: Prednisolone, HD: Hemodialysis, CHDF: Continuous hemodiafiltration, CRP: C-reactive protein, Cre: Creatinine, PIt: Platelet

Although azathioprine was added, the patient developed respiratory failure due to large amounts of pleural effusion that required mechanical ventilation and intrathoracic drainage on day 16. Appropriate antibiotics and antivirals were concurrently administered in anticipation of possible secondary infections. Blood, urine, and pleural fluid cultures were examined as deemed necessary; if infection was considered, antibiotics were administered, or catheter replacements were performed according to infection susceptibility. However, the patient progressed to multi-organ failure. While continuing corticosteroid treatment with these multidisciplinary support modalities, artificial respiration and renal replacement therapy were withdrawn on day 28 and day 52, respectively. Beginning on days 71 and 78 of the corticosteroid treatment, there was a trend of improvement without dialysis in serum Cre level and platelet count, respectively. High inflammatory response with elevated CRP level finally improved, the anasarca disappeared, and the patient was discharged after a total of 104 days of hospitalization. Subsequently, the patient was followed up by a rheumatologist near his home, and the corticosteroid dosage was tapered off until January 2013, for a total of 12 months of corticosteroid treatment. The patient chose not to continue further follow-up hospital visits because his symptoms completely resolved, except for mild Raynaud's phenomenon and sicca syndrome.

In July 2019, seven years and five months after the initial admission, the epigastric pain recurred. In November 2019, he was admitted to our hospital because of worsening epigastric pain and pyrexia. His physical examination revealed abdominal distension, bilateral pitting pedal edema, and a mildly enlarged inguinal lymph node. The laboratory examination results are presented in Table 1. A CT scan revealed splenomegaly, massive ascites, mildly enlarged para-aortal, inguinal, and mediastinal lymph nodes; and pleural effusion (Figures $2 A-2 D$ ). 


\section{Cureus}

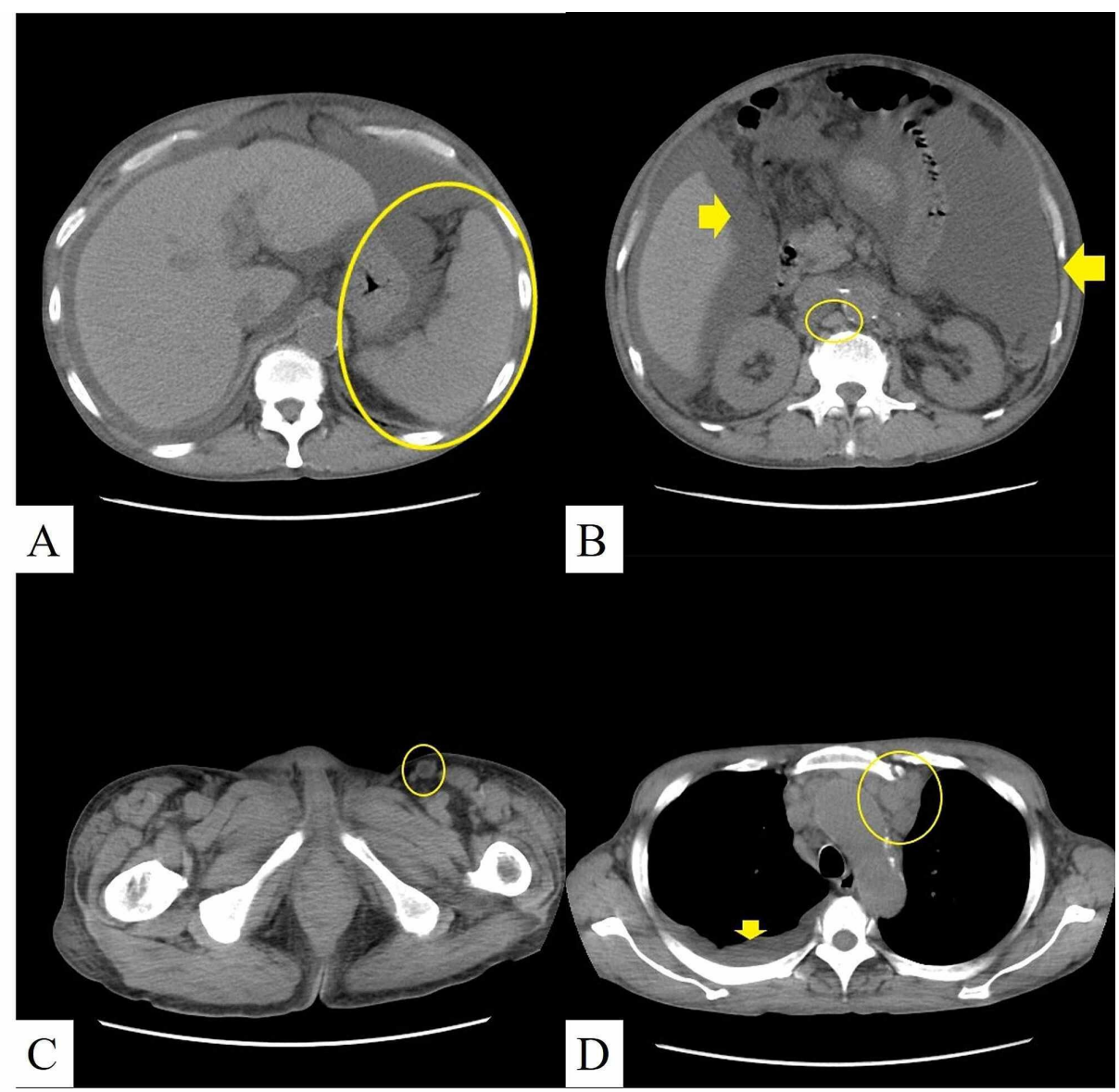

FIGURE 2: A computed tomography scan revealed splenomegaly (circle) (A); massive ascites (arrows); mildly enlarged lymph nodes in the paraaorta (circle) (B); inguinal (circle) (C), and mediastinal regions (circle); and pleural effusion (arrow) (D)

Biopsy specimens from the left inguinal and mediastinal lymph nodes revealed atrophic germinal centers surrounded by increased scattered plasma cells, which were indicative of Castleman disease but made the definitive diagnosis of malignant lymphoma or typical TAFRO syndrome difficult (Figure 3, Panel A).

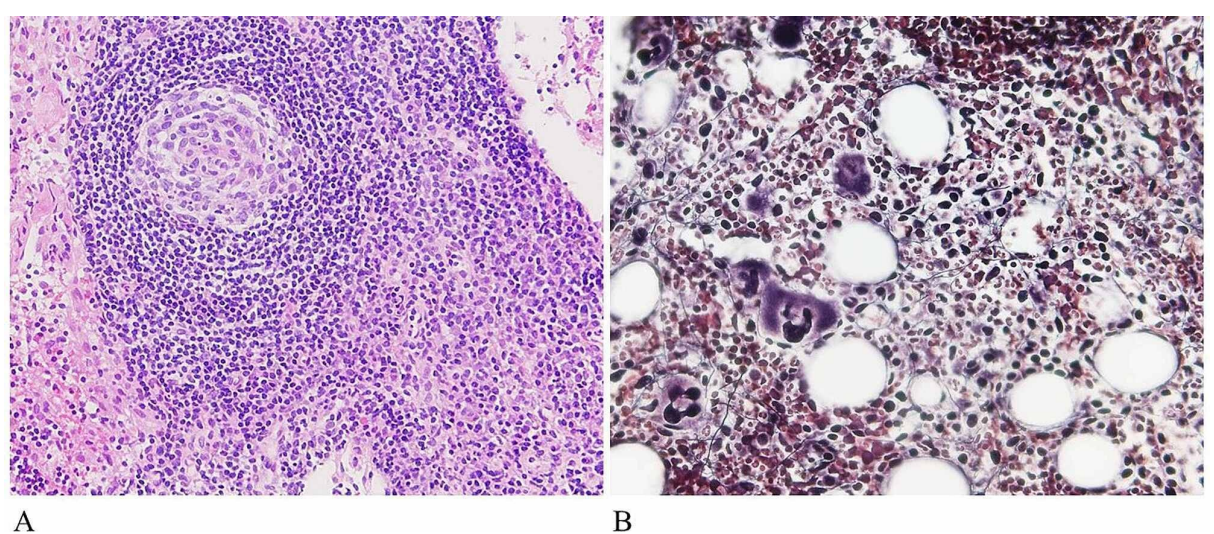

FIGURE 3: Biopsy specimen from the left inguinal lymph node showing an atrophic germinal center surrounded by increased scattered plasma cells (Hematoxylin \& Eosin, $\times 400$ ) (A). Bone marrow biopsy specimen showing an increased number of megakaryocytes and reticulin fibrosis (Gitter staining, ×400) (B) 
Bone marrow biopsy specimens revealed an increase in the number of bone marrow megakaryocytes and reticulin fibers, as shown in Figure 3 (Panel B). His ascites had serous characteristics. No pathogen was detected by culture, and no atypical cells were observed on cytological examination. The clinical symptoms and laboratory findings in November 2019 were like those in February 2012 and mimicked the features of TAFRO syndrome (Tables 1-2 and Figures 2-3).

Years (situation)

2012 (onset)

Findings $\quad$ Thrombocytopenia $\cdot$ anasarca $\cdot$ systemic

resembling inflammation - mild organomegaly · progressive renal

TAFRO insufficiency - elevated level of serum alkaline

syndrome phosphatase

Findings - Raynaud's phenomenon - sicca syndrome with foca unlike lymphocytic sialadenitis from labial salivary gland and

TAFRO low unstimulated whole saliva flow rate $\cdot$ positive for syndrome anti-mitochondrial M2 antibody

\section{9 (relapse)}

- Thrombocytopenia · anasarca · systemic inflammation • mild organomegaly $\cdot$ progressive renal insufficiency • elevated level of serum alkaline phosphatase $\cdot$ beneficial effects of tocilizumab and rituximab

- Raynaud's phenomenon - sicca syndrome - positive for anti-mitochondrial M2 antibody • elevation to hypergammaglobulinemia

TABLE 2: Summary of symptoms during the course of Sjögren syndrome

Therefore, we re-evaluated the patient's symptoms from 2012 because TAFRO syndrome was an unrecognized disorder at that time. However, our findings confirmed a definitive diagnosis of primary Sjögren syndrome based on the classification criteria of the American College of Rheumatology (ACR) and the European League Against Rheumatism (EULAR). In addition, the laboratory abnormalities observed in 2019, including elevated gammaglobulinemia (IgG 2500 (normal range; 861 1747) $\mathrm{mg} / \mathrm{dL}$ ) and positive mitochondrial M2 antibodies are generally found in Sjögren syndrome and not in TAFRO syndrome [11]. Considering that a diagnosis of TAFRO syndrome requires the exclusion of Sjögren syndrome and the absence of hypergammaglobulinemia $[2,8]$, we concluded that his disease was a Sjögren syndrome with TAFRO syndrome-like symptoms. Although cytomegalovirus IgM was positive on admission, the intravenous administration of ganciclovir was ineffective. Thereafter, a high-dose corticosteroid treatment regimen was initiated on day 1 . Pyrexia improved rapidly, but renal dysfunction, ascites, and thrombocytopenia did not respond, as shown in Figure 4.

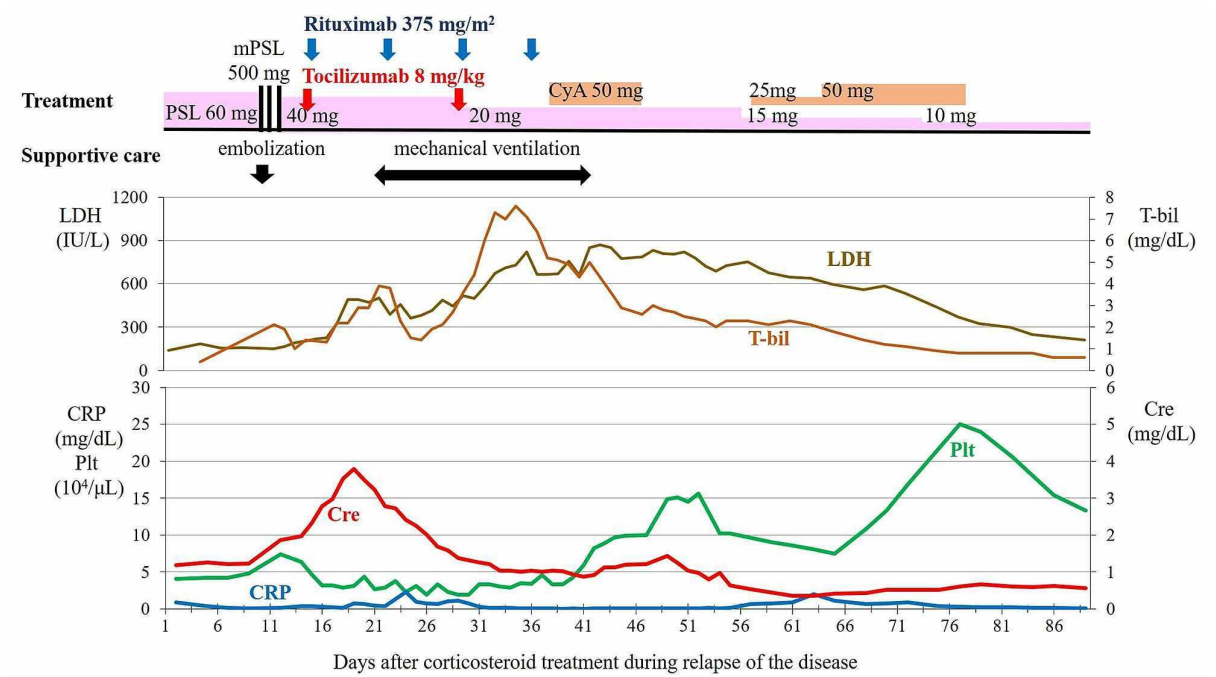

\section{FIGURE 4: Clinical course of relapse}

Abbreviations: mPSL: Methylprednisolone, CyA: Cyclosporine, PSL: Prednisolone, LDH: Lactate dehydrogenase, T-bil: Total bilirubin, Cre: Creatinine, PIt: Platelet, CRP: C-reactive protein

On day 10, he developed mild disorientation and severe abdominal distention, and the characteristic of ascites changed from serous to bloody. The hemoglobin level decreased rapidly, and a contrast CT scan revealed the presence of extravasations with a retroperitoneal hematoma in a large amount of ascites, as 


\section{Cureus}

shown in Figure 5 (Panel A).

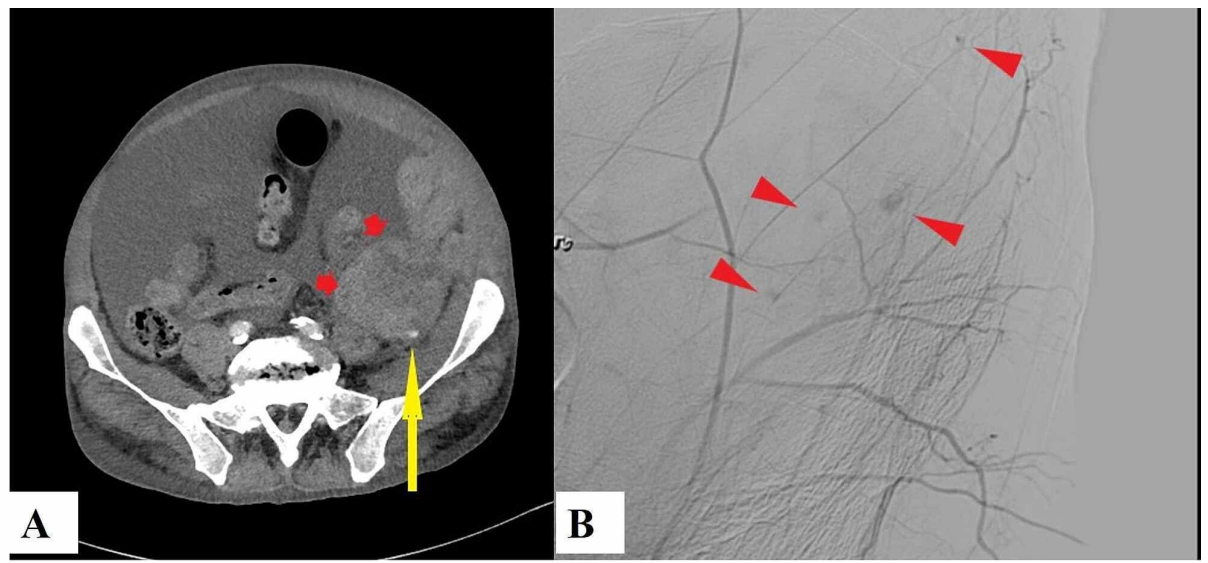

FIGURE 5: Contrast computed tomography scan showing extravasation (long arrow) around the retroperitoneal hematoma (short arrows) (A). Angiographic examination revealed multiple extravasations (arrowheads) in the peripheral vessels (B)

It was clear that the hemorrhage was not caused by the ascites puncture procedures because of the different locations. An emergency angiography revealed multiple microangiopathies of the left peripheral vessels of the iliac lumbar, lumbar, and iliac arteries, which likely caused peritoneal bleeding (Figure 5, Panel B). The angiography revealed no aneurysms or arterial dissections. Although embolization of these bleeding regions was performed and a methylprednisolone pulse (500 mg/day for three days) was started, the hematoma spread into the ascites and the TAFRO symptoms did not improve. At the same time, the platelet count and coagulation examination results were as follows: decreased levels of platelet count $\left(7.4 \times 10^{4} / \mu \mathrm{L}\right)$ and fibrinogen $(137 \mathrm{mg} / \mathrm{dL})$; a slightly elevated level of fibrinogen degradation products $(11.1 \mu \mathrm{g} / \mathrm{mL})$; slightly prolonged prothrombin time (international normalized ratio: 1.26); and a disintegrin and metalloproteinase with a thrombospondin type 1 motif, member 13 (ADAMTS 13), was within the normal range of activity (53\%). Peripheral blood smears did not show remarkable fragmentation of the red blood cells. The patient was in a critical condition with progressive renal dysfunction, making adequate use of immunosuppressive drugs, such as cyclosporine and azathioprine, difficult. After written informed consent was obtained from the patient and his wife, additional treatment with intravenous tocilizumab ( $8 \mathrm{mg} / \mathrm{kg}$, once every two weeks beginning from day 14) and rituximab (375 mg/m², once a week from day 15), without the need to adjust the dose due to renal dysfunction, was initiated in addition to prednisolone based on the proposed treatment for TAFRO syndrome [1]. His consciousness progressively worsened from day 17 . In parallel, there were gradual increases in serum total bilirubin (T-bil) (indirect bilirubin dominant) and lactate dehydrogenase (LDH) levels and decreases in haptoglobin level $(<10 \mathrm{mg} / \mathrm{dL})$, which indicated a new complication of hemolytic anemia as shown in Figure 4. The patient's renal dysfunction, thrombocytopenia, and microangiopathy were accompanied by a disturbance in the level of consciousness, hemolytic anemia, and LDH elevation, suggesting the development of TMA. Thereafter, he developed severe aspiration pneumonia against a background of confusion and required a mechanical ventilator on day 21 . With repeated administration of tocilizumab and rituximab plus the continuation of corticosteroid and supportive treatment, serum Cre showed a trend of improvement from day 19, T-bil from day 35, and platelets from day 39. Similarly, his breathing improved, and he was able to discontinue ventilatory support on day 41 . Anemia also improved and red blood cell transfusions were no longer needed from day 31. Although the amount of ascites was markedly reduced, the hematoma with a small amount of ascites remained. Similarly, the lymph node enlargement was reduced but did not disappear. The tapered prednisolone normalized laboratory serum data, such as the platelet count, Cre, CRP, T-bil, and LDH. The patient was doing well and was rehabilitated and discharged home after a total of 102 days in our hospital. During the 10 months outpatient follow-up period, the patient experienced no symptom recurrence with $5 \mathrm{mg}$ of oral prednisolone.

\section{Discussion}

The symptoms in this present case closely resembled TAFRO syndrome in terms of clinicopathological findings, aggressive clinical course, and beneficial effects of tocilizumab and rituximab [1-2,6-7,12]. However, according to the diagnostic criteria for TAFRO syndrome proposed by Masaki et al., TAFRO syndrome can only be diagnosed after excluding Sjögren syndrome, and the thrombocytopenia, anasarca, fever, myelofibrosis, organomegaly, and renal insufficiency in this patient can be accounted for by Sjögren syndrome $[2,11,13-14]$. Similarly, according to the diagnostic criteria proposed by Iwaki et al., the absence of hypergammaglobulinemia is a major criterion for the diagnosis of TAFRO-iMCD. Therefore, we concluded 
that this patient was diagnosed as having Sjögren syndrome with TAFRO syndrome-like symptoms. There are previous case reports and studies of patients with a definitive diagnosis of Sjögren syndrome or those positive for anti-SS-A and/or anti-SS-B antibodies but with an uncertain diagnosis that developed clinical features of TAFRO syndrome, and some of them relapsed [3-7]. In this case, TAFRO syndrome-like symptoms, such as thrombocytopenia, anasarca, fever, myelofibrosis, organomegaly, and renal insufficiency also occurred with Sjögren syndrome. These results suggest that the etiology of TAFRO syndrome may be associated with Sjögren syndrome. Furthermore, TAFRO syndrome may co-exist with Sjögren syndrome. More recently, increased serum interferon $\gamma$-induced protein 10 (IP-10), a cytokine belonging to the CXC chemokine family, was observed in patients with TAFRO syndrome during flare-ups [15]. Considering that the expression of IP-10 has been associated with autoimmune diseases, including Sjögren syndrome; inflammatory diseases, including viral, bacterial, and mycotic infections; immune dysfunction; and tumorigenesis; TAFRO syndrome develops in association with these diseases. Interestingly, in this case, the levels of CRP at relapse were not increased as compared to the onset during Sjögren syndrome, as shown in Table 1, Figure 1, and Figure 4. Hence, we hypothesize that the TAFRO symptoms in this patient may be caused by different IP-10-expressing factors. For example, we can assume that the trigger at onset might be a bacterial infection and a trigger at relapse might be a viral infection, resulting in different CRP levels with a background of Sjögren syndrome. The etiology of TAFRO syndrome and the association between Sjögren syndrome and TAFRO syndrome are still unclear. It is expected that biomarkers specific to TAFRO syndrome will be identified in the future.

In this case, the retroperitoneal hemorrhage may have been related to TMA, although other causes, such as vascular weakness, thrombocytopenia, and disseminated intravascular coagulation, might have also been related. The most common autoimmune disorders complicating secondary TMA are systemic lupus erythematosus and systemic sclerosis; there are also reports of Sjögren syndrome complicating TMA [16-17]. In TAFRO syndrome, renal biopsies often show TMA-like findings that may be crucial to the development of acute renal dysfunction [5]. Additionally, tocilizumab was also reported to cause an adverse event of multifocal microangiopathy [18]. The bleeding and disorders of consciousness, in this case, occurred prior to the first infusion of tocilizumab, suggesting that tocilizumab was not the primary cause of TMA. Secondary TMA has been reported to be caused by a variety of triggers, including autoimmune diseases, infections, drugs, malignancies, transplants, and pregnancy [19]. Therefore, it is difficult to identify a specific factor, and this case may have involved multiple factors, including Sjögren syndrome, TAFRO symptoms, tocilizumab, as well as others.

The patient's relapse symptoms recovered earlier and were less severe than those at onset (Figure 1 and Figure 4). We considered this amelioration of symptoms to be the result of the early diagnosis of relapse and rapid therapeutic intervention, the previously mentioned different triggers hypotheses, and the effectiveness of additional therapies with tocilizumab and rituximab. Recently, tocilizumab or rituximab for primary Sjögren syndrome has failed to show significant advantages in randomized double-blind controlled trials, although these assessed outcomes were different from TAFRO symptoms [20]. On the contrary, tocilizumab, rituximab, or both have been reported as effective and well-tolerated in case reports of TAFRO syndrome and have been recommended as a treatment option [1,4-7,12]. A standard regimen for the order, methods, and duration of the administration of tocilizumab and rituximab has not been established. However, the coadministration of tocilizumab, rituximab, and corticosteroid has been used in first-line treatment, in relapse, and in critical situations, and successful responses to the treatment have been reported [5,12]. This presented case was in a recurrent, corticosteroid-resistant, and life-threatening situation. Furthermore, because renal replacement therapy using anticoagulants was not feasible due to the risk of worsening retroperitoneal hemorrhage, we decided to use coadministration therapy, expecting an immediate therapeutic effect. As a result, the coadministration therapy at relapse resulted in early response and recovery of TAFRO symptoms as compared to the symptoms at the onset. In addition, after these therapies, the progression of retroperitoneal hemorrhage stopped. Blood examinations, including T-bil and $\mathrm{LDH}$, eventually normalized. Coadministration therapy using tocilizumab, rituximab, and corticosteroid may have been effective for hemorrhage probably due to TMA as well as TAFRO syndrome-like symptoms during Sjögren syndrome.

\section{Conclusions}

The TAFRO syndrome-like symptoms during Sjögren syndrome closely resembled TAFRO syndrome in terms of the clinicopathological findings, aggressive clinical course, and beneficial effects of tocilizumab and rituximab. The etiology of TAFRO syndrome could potentially involve Sjögren syndrome, and these syndromes may be co-existing. A careful follow-up of Sjögren syndrome would be necessary because lifethreatening TAFRO symptoms may recur and lead to vascular complications related to TMA. Accumulation of similar cases and further research are needed.

\section{Additional Information \\ Disclosures}

Human subjects: Consent was obtained by all participants in this study. Conflicts of interest: In compliance with the ICMJE uniform disclosure form, all authors declare the following: Payment/services info: All authors have declared that no financial support was received from any organization for the 
submitted work. Financial relationships: All authors have declared that they have no financial relationships at present or within the previous three years with any organizations that might have an interest in the submitted work. Other relationships: All authors have declared that there are no other relationships or activities that could appear to have influenced the submitted work.

\section{Acknowledgements}

We thank Dr. Kakihara and Dr. Nakamura, who treated with urgent angiography and embolization, and the nursing staff who cared for the patient at Kitakyushu Municipal Medical Center. We would like to thank Editage (www.editage.com) for English language editing.

\section{References}

1. Masaki Y, Kawabata H, Takai K, et al.: Proposed diagnostic criteria, disease severity classification and treatment strategy for TAFRO syndrome, 2015 version. Int J Hematol. 2016, 103:686-692. 10.1007/s12185016-1979-1

2. Masaki Y, Kawabata H, Takai K, et al.: 2019 updated diagnostic criteria and disease severity classification for TAFRO syndrome. Int J Hematol. 2020, 111:155-158. 10.1007/s12185-019-02780-1

3. Fujimoto S, Kawabata H, Kurose N, et al.: Sjögren's syndrome manifesting as clinicopathological features of TAFRO syndrome. A case report. Medicine (Baltimore). 2017, 96:e9220. 10.1097/MD.0000000000009220

4. Li ZY, Kim S, Huang S, Mian R: Multicentric Castleman disease with TAFRO syndrome and Sjögren's. Clin Case Rep. 2019, 7:2388-2392. 10.1002/ccr3.2502

5. Louis C, Vijgen S, Samii K, et al.: TAFRO syndrome in Caucasians: a case report and review of the literature . Front Med. 2017, 4:149. 10.3389/fmed.2017.00149

6. Tsurumi H, Fujigaki Y, Yamamoto T, et al.: Remission of refractory ascites and discontinuation of hemodialysis after additional rituximab to long-term glucocorticoid therapy in a patient with TAFRO syndrome. Intern Med. 2018, 57:1433-1438. 10.2169/internalmedicine.0116-17

7. Kikuchi T, Shimizu T, Toyama T, Abe R, Okamoto S: Successful treatment of TAFRO syndrome with tocilizumab, prednisone, and cyclophosphamide. Intern Med. 2017, 56:2205-2211. 10.2169/internalmedicine.8522-16

8. Iwaki N, Fajgenbaum DC, Nabel CS, et al.: Clinicopathologic analysis of TAFRO syndrome demonstrates a distinct subtype of HHV-8-negative multicentric Castleman disease. Am J Hematol. 2016, 91:220-226. 10.1002/ajh.24242

9. Fajgenbaum DC, Uldrick TS, Bagg A, et al.: International, evidence-based consensus diagnostic criteria for HHV-8-negative/idiopathic multicentric Castleman disease. Blood. 2017, 129:1646-1657. 10.1182/blood2016-10-746933

10. Liu AY, Nabel CS, Finkelman BS, et al.: Idiopathic multicentric Castleman's disease: a systematic literature review. Lancet Haematol. 2016, 3:163-175. 10.1016/S2352-3026(16)00006-5

11. Lin DF, Yan SM, Zhao Y, et al.: Clinical and prognostic characteristics of 573 cases of primary Sjögren's syndrome [Article in Chinese]. Chin Med J. 2010, 123:3252-3267. 10.3760/cma.j.issn.0366-6999.2010.22.015

12. José FF, Kerbauy LN, Perini GF, et al.: A life-threatening case of TAFRO syndrome with dramatic response to tocilizumab, rituximab, and pulse steroids. The first case report in Latin America. Medicine (Baltimore). 2017, 96:e6271. 10.1097/MD.0000000000006271

13. Rizzi R, Pastore D, Liso A, et al.: Autoimmune myelofibrosis: report of three cases and review of the literature. Leuk Lymphoma. 2004, 45:561-566. 10.1080/10428190310001597982

14. Liu Y, Chen S, Sun Y, et al.: Clinical characteristics of immune thrombocytopenia associated with autoimmune disease. A retrospective study. Medicine (Baltimore). 2016, 95:e5565. 10.1097/MD.0000000000005565

15. Iwaki N, Gion Y, Kondo E, et al.: Elevated serum interferon $\mathrm{Y}$-induced protein $10 \mathrm{kDa}$ is associated with TAFRO syndrome. Sci Rep. 2017, 7:42316. 10.1038/srep42316

16. Cheng MH, Lin JH, Yen TH, et al.: Thrombotic microangiopathy complicating newly diagnosed Sjögren's syndrome in a dialysis patient. Ren Fail. 2014, 36:1162-1165. 10.3109/0886022X.2014.917764

17. Matsuyama T, Kuwana M, Matsumoto M, Isonishi A, Inokuma S, Fujimura Y: Heterogeneous pathogenic processes of thrombotic microangiopathies in patients with connective tissue diseases. Thromb Haemost. 2009, 102:371-378. 10.1160/TH08-12-0825

18. Jewell P, Ansorge O, Kuker W, Irani SR, Zamboni G: Tocilizumab-associated multifocal cerebral thrombotic microangiopathy. Neurol Clin Pract. 2016, 6:e24-e26. 10.1212/CPJ.0000000000000220

19. Campistol JM, Arias M, Ariceta G, et al.: An update for atypical haemolytic uraemic syndrome: diagnosis and treatment. A consensus document [Article in Spanish]. Nefrologia. 2015, 35:421-447. 10.1016/j.nefro.2015.07.005

20. Mavragani CP, Moutsopoulos HM: Sjögren's syndrome: old and new therapeutic targets. J Autoimmun. 2020, 110:102364. 10.1016/j.jaut.2019.102364 